\title{
LA QUESTION DES DROITS HUMAINS COMME PRECEPTES OCCIDENTAUX
}

THE ISSUE OF HUMAN RIGHTS AS OCCIDENTAL PRECEPTS

Vivianny Galvão

Professeur à la Faculté de Droit de l'UNIT. Doctorat en Sciences Juridiques (UFPB). Master 2 en Droit Public (UFAL) Chercheuse au Laboratoire de Droits de l'Homme (UFAL)s. viviannygalvao@hotmail.com

Résumé: Où nait la force des droits humains ? Les droits humains qui devront prévaloir représenteront-ils toujours la protection d'un attribut universel présent en tout être humain ? Ou bien ces standards devront-ils dépendre de la culture ou du groupe social de la personne humaine ? Tandis que certains manifestent la préoccupation concernant la limitation des recours à la critique et à la justification de la diversité des codes moraux, d'autres jugent illégitime (et improbable) la recherche de normes extensives à tous - qui que ce soit, où que ce soit. Les normes morales ne s'acquièrent qu'au sein de la structure sociale, autrement dit, dans l'ensemble particulier des restrictions physiques et sociales ? Et les raisons qu'elles ont pour justifier leurs normes ne seront-elles impositives qu'à ceux qui partagent la même condition ? Nombreuses sont les questions concernant l'étendue des droits humains. La reconnaissance légale est condition nécessaire, mais non suffisante, à la pleine jouissance des droits. Même ainsi, de nos jours il n'est pas nécessaire de recourir à des arguments moraux comme uniques raisons pour dénoncer l'illégitimité de quelque législation et de certaines décisions d'État. L'ouverture à des discussions relatives aux meilleures méthodes pour organiser les sociétés politiques et civiles est le signe essentiel à la promulgation légale des droits humains. La méthodologie utilisée était quantitative, la méthode d'approche inductive, la méthode de procédure historique, parmi les plus importants.

Mots-clés: Condition de la personne humaine - Droits humains - Société internationale.

Abstract: Where the strength of human rights comes? Human rights which should avail they still represent the protection of a universal 
attribute present in every human being? Either these standards they will depend on the culture or social group of human beings? While some express concern regarding the limitation of use of criticism and justification of the diversity of moral codes, others deem illegitimate (and unlikely) the extensive research standards at all - anyone, anywhere either. Moral standards can only be learned that within the social structure, that is, in the particular set of physical and social restrictions? And the reasons they have to justify their standards will they impositives only those who share the same condition? There are many questions about the extent of human rights. Legal recognition is necessary, but not sufficient, to the full enjoyment of rights. Even so, these days it is not necessary to resort to moral arguments as unique reasons to denounce the illegitimacy of some laws and some state decisions. The opening of discussions on best ways to organize political and civil societies is the essential sign the legal enactment of human rights. The methodology used was quantitative, inductive approach method, historical method of procedure, among the most important.

Keywords: Condition of the human being - Human rights - International society

\section{INTRODUCTION}

L'expression «droits humains » est très employée. Elle est sortie des salles universitaires, a dépassé les décisions judiciaires et a gagné les rues. Dans chacun de ces espaces elle a acquit un sens propre. C'est pourquoi la proximité de l'abordage historiciste - entrepris ici sans la moindre prétention - passe par la mention de faits et concepts et renforce l'intention de ne pas confondre les éléments et leurs espaces. Dans les sciences sociales, beaucoup d'auteurs ont étudié ce qu'il est convenu d'appeler " droits humains ». Dans les diverses formes de libéralisme, dans les diverses lignes de pensée socialiste, dans les écrits philosophiques, sociologiques, économiques, juridiques etc, tous ont leur concept de droits humains.

Pour ce qui est du rapport entre droits humains et droits fondamentaux, on sait qu'ils sont tous deux liés à la notion de droit à quelque chose (ALEXY, 2003, p. 16-20). Aussi bien les droits fondamentaux que les droits humains sont garantis par des ordres juridiques. En raison de la pression entre les diverses forces sociales, les droits fondamentaux se présentent comme un ensemble de valeurs objectives de base et comme jalon de protection des situations juridiques subjectives (PÉREZ LUÑO, 2004, p. 43-46). Il est possible de trouver des droits positivés comme fondamentaux dans un ordre d'État déterminé qui ne représentent pas des droits humains reconnus 
dans l'ordre juridique international. Bref, les droits fondamentaux sont essentiellement des droits de la personne humaine et représentants des droits humains transformés en droit constitutionnel positif (ALEXY, 2003, p. 21-29).

Il y a aussi la question de l'établissement des droits humains. Pour Eusebio Fernández (1981, p. 77), la recherche des fondements des droits humains touche au problème de les justifier rationnellement. L'auteur relève trois fondements : a) jusnaturaliste (droits naturels) ; b) historiciste (droits historiques) ; et c) éthique (droits moraux). Dans la justification jusnaturaliste, le droit naturel naît de la nature humaine et compose l'ordonnance universelle. Ce droit sera toujours au-dessus du droit positivé (FERNÁNDEZ, 1981, p. 80). Jean Morange (1982, p. 45) reconnaît (1) le droit naturel objectif, développé par Aristote, considéré effet de la Nature, révélateur de Dieu créateur et obéissant à l'ordre rationnel des choses; et (2) le droit naturel subjectif, qui chez Platon découle de la Nature de l'être humain, car chaque être posséderait quelque chose de divin en soi. Les droits humains, sont'ils preceptes occidentaux?

\section{LES FONDEMENTS DES DROITS HUMAINS}

Sur toutes les théories des droits naturels retombent les critiques suivantes : a) les droits humains ne peuvent être supérieurs ni antérieurs au droit positif puisqu'ils n'ont été positivés en aucun ordre ; b) l'idée de nature humaine serait encore profondément ambiguë ; c) la notion de droits naturels immuables entre en choc avec l'expérience historique (FERNÁNDEZ, 1981, p. 88). L'utilitarisme de Bentham, qui a dominé la pensée sociale anglaise une grande partie du XIXe siècle, renforce l'idée de contradiction dans l'existence de droits antérieurs au droit positif (HART, 1981, p. 149-168). En dépit des critiques véhémentes, les réflexions sur le droit naturel n'ont pas disparues au XIXe siècle. Leo Strauss, par exemple, reprend le débat et défend que la décadence de la philosophie politique est liée à la rupture d'avec l'idée de droits naturels classiques (1986, p. 99).

Dans la raison historiciste, les droits humains sont historiques, variables, relatifs. Il s'agit de droits d'origine sociale. La principale critique faite à l'historicisme est la perception selon laquelle quelques droits ne seraient pas aussi variables. Il serait possible de défendre l'existence relative au moment historique de droits civiques et politiques, économiques et sociaux, culturels. Toutefois, pourrait-on en dire autant des droits personnels, du droit à la vie et à l'intégrité physique et morale ? De plus, ce fondement historiciste entrave toute construction de droits fondamentaux. Face à cette critique, Eusebio Fernández souligne la nécessité de distinguer la vision historique des droits humains de leur 
argumentation historiciste (FERNÁNDEZ, 1981, p. 94).

Quant a l'argumentation éthique, elle considère les droits humains comme étant des droits moraux. Ici, les droits humains assument deux versants indissociables : éthique et juridique. Les droits humains sont moraux parce qu'ils sont strictement liés à l'idée de dignité humaine (FERNÁNDEZ, 1981, p. 98-99). Ils apparaissent comme exigences éthiques et comme droits que les êtres humains possèdent par le fait qu'il s'agit d'êtres humains. En outre, ils imposent au Pouvoir Politique et au Droit (sens objectif) leur reconnaissance, protection et garantie. La dignité humaine fonctionne comme critère de vérification des systèmes éthiques qui doivent placer au premier plan la satisfaction des nécessités humaines, le développement des capacités personnelle, l'élimination des souffrances et la concrétisation des désirs.

L'argumentation des droits humains dans la conception marxiste (matérialiste) les désigne comme étant une conquête historique de la bourgeoisie. Bien qu'il n'y ait pas de théorie juridique, quand Marx théorise l'extinction de l'Etat, se trouve implicite dans ses écrits la théorisation de l'extinction de la forme juridique. Sous cet angle, il est possible d'analyser les droits humains dans la théorie marxiste. Manuel Atienza Rodríguez relève une certaine ambiguïté chez Marx par rapport aux droits humains. Il dit que, bien qu' étant critique des droits humains, Marx s'est rendu compte que la grande contradiction existante entre ces derniers et le système capitaliste pourrait également être là où ce système s'écroulerait. Ainsi, les droits humains ne seraient jamais une fin en soi valorisée éthiquement, mais plutôt des instruments politiques (ATIENZA, 2008, p. 226).

Dans cette recherche ou réfutation des fondements des droits humains, émergent d'autres questions. Le rapport anachronique entre positivité des droits humains et leur effectivité sociale, le rôle des sujets et des acteurs de droit international, l'efficacité des normes du droit international des droits humains et leur étendue etc. Pour cette raison, la compréhension historique des droits humains doit contribuer moyennant des propositions plus critiques aux problèmes présentés.

\section{Retour AU DÉbat ENTRE JELLINEK ET BOUTMY : CONNAISSANCE DE LA PENSÉE OCCIDENTALE DES DROITS HUMAINS}

La philosophie des droits humains naît à la fin du XVIIIe siècle, en Europe Occidentale et en Amérique du Nord. Sa présence s'est prêtée à la composition de " l'esprit de l'époque », dans la guerre idéologique au service d'une classe sociale déterminée. Les droits humains étaient vus comme le résultat d'une évolution purement rationnelle dont nul ne pouvait s'écarter (MORANGE, 1982, p. 27). Selon Georg Jellinek, la naissance de la philosophie des droits humains coincide avec les 
Déclarations des Droits. Le texte qui a suscité le débat avec Émile Boutmy, La Declaración de los Derechos del Hombre y del Ciudadano, analyse le document le plus important de la Révolution Française, la Déclaration des Droits de l'Homme et du Citoyen, du 26 août 1789. C'est à partir de ce document que l'on peut parler de « droits humains ». Ainsi, quelle est l'origine de la Déclaration des Droits de l'Homme et du Citoyen (ou Déclaration Française de 1789)?

Selon Jellinek, la Déclaration Française de 1789 révèle des aspects politiques et historiques, ainsi que historico-juridiques. Avant la Déclaration Française, la littérature juridico-politique ne connaissait que les droits des Chefs d'Etat, les privilèges de classe, de particuliers ou des corporations. La Déclaration a influencé les Constitutions Françaises du 3 septembre 1791 et du 4 novembre 1848 quant à l'insertion de catégories juridiques reconnues aux personnes (auparavant connues uniquement par le Droit Naturel). Jusqu'en 1848, la plupart des Constitutions Allemandes parlaient de droit des sujets; à partir de cette date, l'Assemblée votait les droits fondamentaux du peuple allemand (JELLINEK, 2000, p. 42). Il s'est produit une reproduction à grande échelle de ces catégories.

Jellinek critique l'abordage superficiel des recherches sur l'origine de la Déclaration Française de 1789. Pour lui, les ouvrages de Droit Politique se sont contentés de relever les précédents de la Déclaration (de la Grande Charte jusqu'á la Déclaration d'Indépendance américaine), sans enquêter avec une plus grande profondeur les sources qui ont inspiré les Français et qui, en d'autres termes, ont donné naissance à la philosophie des droits humains. La théorie du contrat social (comme fondement de la Déclaration Française) et la Déclaration d'Indépendance des 13 États Unis d'Amérique du Nord (comme modèle de la Déclaration Française) constituent les questions de fond (JELLINEK, 2000. P. 41-44).

Paul Janet, dans son História da ciência política, affirme l'influence du contrat social sur la Révolution Française. Jellinek critique cette affirmation parce que, pour lui, Rousseau n'a fait que présenter un principe dans le contrat social : le remplacement de tous les droits de l'individu à la société. Tout droit découlerait de la volonté générale. Devant cela, aucun droit individuel ne se conserverait en entrant dans l'État. Toute liberté civile correspondant aux devoirs civiques se perdrait. L'idée de droit originaire transféré à la société pour limiter juridiquement le souverain n'existerait pas. Il n'existerait que des libertés contraires à l'État (liberté de religion, droit d'association). Les principes du contrat social ne naitraient pas de droits individuels, mais de l'omniprésence de la volonté générale. Par conséquent, rien ne serait plus contraire à la Déclaration que la base du contrat social de Rousseau.

Sans nier l'influence du contrat social (JELLINEK, 2000, p. 67) 
sur la Déclaration Française - qu'il signale dans les articles 4, 6 et 13 -, il passe à l'examen des Bills of rights des États Particuliers de l'Union Nord-Américaine. Pour Jellinek, dans les archives parlementaires françaises, il y avait déjà un chapitre qui traitait de la nécessité d'établir des droits au peuple et c'est le Marquis de Lafayette qui a présenté cette proposition à l'Assemblée Nationale le 2 juillet 1789. Bien que Lafayette, aristocrate français, ait participé à la Guerre d'Indépendance des ÉtatsUnis d'Amérique et au début de la Révolution Française, selon les mémoires du Marquis, la Déclaration d'Indépendance n'a formulé que des principes de souveraineté nationale et des droits pour le changement de la forme de gouvernement. (JELLINEK, 2000, p. 49-53).

À propos des mouvements constitutionnels antérieurs à la Révolution Française, Jellinek affirme que les Constitutions des États Particuliers de l'Union étaient précédées de Déclarations de Droits, la première en date étant la Déclaration de Virginie (JELLINEK, 2000, p. 51). Le 15 mai 1776, le Congrès de Philadelphie représentait les colonies qui voulaient leur séparation d'avec la Couronne Britannique. Des 13 colonies, 11 avaient déjà adhéré à la rupture, tandis que deux avaient transformé les chartes coloniales octroyées en Constitutions (Charte de Connecticut de 1662 et de Rhode Island de 1663). L'État de Virginie a été le premier à adopter une constitution avec un Bill of rights, entre le 6 et 29 juin 1776, à la Convention de Williamburg. Elle a influencé les autres Constitutions et le Congrès des États-Unis (il mentionne que Jefferson, citoyen de Virginie, en fut le rédacteur). Ainsi, l'auteur admet-il l'influence de la Déclaration de Virginie dans le modèle adopté par la Déclaration Française de 1789.

Quant aux Déclarations anglaises, elles n'ont pas eu autant d'impact sur le modèle adopté par les révolutionnaires français. Pour Jellinek, autant la Déclaration française que les Déclarations américaines ont énoncé avec la même passion des principes abstraits. Toutefois, la Déclaration française, en adoptant le modèle américain, serait restée en deçà de celui-ci, car elle ne le surpasse en contenu que lorsqu'elle traite, timidement, dans l'article 10, des manifestations d'opinions en matière religieuse. Même comme ça, elle se contente de proclamer la tolérance, et non pas la liberté religieuse. Aux ÉtatsUnis, des communautés organisées se sont créées ; en France, c'est la perturbation sociale qu'elle a engendrée (Lalley Tollendal et Mirabeau) (JELLINEK, 2000, p. 67-70).

Il ne resterait guère aux textes anglais (Bill of Right de 1689, Habeas Corpus de 1679, Petition of Rights de 1627 et la Magna Charta Libertatum de 1215) la formation des fondements des Bills of rights américains. Outre ce laps de temps, Jellinek souligne (se fondant sur Baneroft - historien de la Révolution Américaine - et Sir Edward Coke - jurisconsulte anglais) que les lois anglaises étaient purement 
historiques, ponctuelles et qu'elles n'avaient nullement l'intention de reconnaître des droits généraux « de l'homme ». Toute loi élaborée et acceptée par le Parlement possédait une égale valeur et il n'y avait pas de différence entre les législateurs constitutionnels et ordinaires. Les Bills of rights américains déterminaient la ligne de séparation entre les individus et l'État, tandis que les lois anglaises traitaient des devoirs du Gouvernement (JELLINEK, 2000, p. 71). Seuls deux (des treize) points se rapportaient aux droits des sujets. Les droits du peuple se résumaient à l'idée de restrictions imposées à la Couronne (conception médiévale - Ve et Xve siècles - visible dans l'État germanique, où le peuple et le prince, parce qu'étant opposés et indépendants, auraient besoin d'établir une relation contractuelle). Les lois anglaises ne traitaient que d'anciens droits et libertés.

L'idée selon laquelle les droits à la liberté sont des devoirs du Gouvernement se serait développée en Grande-Bretagne, principalement avec l'affaiblissement de la doctrine de Locke et de Blackstone. Georg Jellinek trouve le droit à la liberté dans l'idée de Locke sur la propriété. Pour Locke, la propriété est un droit originaire antérieur à l'État, c'est pourquoi il incombe à ce dernier de la protéger. Cependant, ce droit à la liberté ne serait rien de plus qu'une attribution limitatrice du Pouvoir Législatif. Quant à Blackstone, il reconnaissait aux sujets anglais l'exercice de la sécurité, de la liberté et de la propriété, qui se fondait sur la liberté naturelle, dispensant toute restriction légale au nom de l'intérêt commun. Les Déclarations américaines reconnaissent une liste bien plus grande de droits innés et inaliénables à tous dès la naissance. Puisque le modèle adopté par les Déclarations américaines ne viendrait pas des lois anglaises, Jellinek revient sur les conceptions de droit naturel de l'époque. Auparavant, il rappelle que les anciennes conceptions de droit naturel n'avaient pas été développées pour être confrontées au droit positivé (ex : Ulpiano visualisait l'égalité des hommes par le droit naturel et acceptait l'esclavage comme institut du droit civil, ainsi que Locke dans la Constitution de la Caroline du Nord) (JELLINEK, 2000, p. 77-79).

L'origine des droits universels « de l'homme » résiderait dans la liberté religieuse des colonies anglo-américaines, spécialement dans le Congrégationisme de Roberto Brown - fin du XVIe siècle en Angleterre -, origine de la forme primitive de l'Indépendantisme, Il s'agit de l'idée de séparation entre l'Église et l'État, ainsi que de 1'autonomie de chaque communauté. Le coup d'envoi, en Angleterre, du développement de cette pensée a été la soumission de l'agreement of the people au Conseil Général de l'armée de Cromwell le 28 octobre 1647. L'agreement, transformé en projet et présenté au Parlement anglais, contenait la proposition de limiter le Parlement et de laisser à la charge des connsciences les questions religieuses (JELLINEK, 2000, p. 79). 
Ces « pactes d'établissement » ont été réalisés par les pasteurs pèlerins congrégationistes dans la fondation des colonies anglaises du nouveau monde, créant des contrats en conformité avec leurs principes ecclésiastiques et politiques. Ces pactes reconnaissaient et garantissaient la liberté religieuse. Ils ont été célébrés aussi bien à Salem, fondée par des puritains en 1629, Massachusetts, qu'à Providence, fondée en 1636 par Roger Williams, sous couvert de l'idéal selon lequel « la conscience de l'homme appartient à lui-même, et non pas à l'Etat ». D'une façon ou d'une autre, les pactes ne réglementaient que des affaires civiles. De là serait apparue naturellement la forme de démocratie directe (JELLINEK, 2000, p. 80).

La liberté religieuse absolue cherchée par Roger Williams n'a été reconnue officiellement qu'à travers le Code de Rhode Island (1647) et la Charte (1663) que Charles II a octroyé aux colonies de Rhode Island et aux Plantations de Providence. L'Europe ne vivrait quelque chose de semblable qu'avec les Maximes de Frédéric de Prusse, lors de son avènement au trône en 1740. Le principe de la liberté religieuse aurait atteint en Amérique une consécration juridico-constitutionnelle. Le droit à la liberté de conscience ouvrait une voie à la naissance d'un " droit de l'homme ». Jellinek observe que l'idée de positiver de tels droits n'a pas été politique, mais bien religieuse. L'auteur voit en Roger Williams, et non pas en Lafayette, le premier apôtre des « droits de l'homme » (JELLINEK, 2000, p. 80-86).

La force des événements historiques a aidé à mettre en relief les théories du droit naturel. Jellinek critique l'abstraction de ces droits et signale l'exigence d'un relevé de droits fondamentaux reconnus expressément par l'État et à partir de la Révolution américaine. Avec le développement économique des colonies, sont apparues d'autres mesures essayant de les restreindre, même devant la reconnaissance des américains comme citoyens anglais. À ce moment-là, existait déjà l'idée de liberté de conscience, ainsi que la reconnaissance du fait que les personnes conservent en société leurs droits et que ces droits devaient être considérés dans l'État et contre l'État (JELLINEK, 2000, p. 87-89).

Les tentatives de limiter les colonies ont impulsé le mouvement de déclaration de ces droits. Les déclarations reconnaissaient des droits de liberté personnelle, de propriété, de conscience, des droits de libertés individuelles (presse, réunion, établissement), ainsi que des droits de pétition, protection légale, procédure judiciaire applicable et garanties politiques ;en général, droits publics des individus. Les textes prévoyaient aussi le principe de séparation des pouvoirs et de la responsabilité des fonctionnaires, la temporalité de l'occupation des charges et les limites à leur exercice. La souveraineté est du peuple, et la Constitution doit être formulée par tous. Jellinek signale les contradictions dans l'emploi des termes man et freeman, au lieu de citizen. Les termes originaux 
donnaient de la marge au déni d'humanité de certains groupes humains (par la race, le genre etc) (JELLINEK, 2000, p. 96).

La réponse d'Émile Boutmy à la thèse soutenue par Jellinek à propos de la contradiction entre les Déclarations de droit et les principes du Contrat social mérite d'être prise en considération (Boutmy, 1907, p. 122). Il défend que la philosophie de Rousseau et les maximes du Contrat social ont pu influencer en partie les articles de la Déclaration Française de 1789. Selon Boutmy, la Déclaration n'est aucunement contradictoire avec les principes du Contrat social. Le Contrat social représenterait la convention entre deux personnages, l'un abstrait (la totalité des individus) et l'autre concret (l'unanimité d'individus considérés isolément). Les conséquences du Contrat seraient la constitution d'un corps politique, composé par l'État (ou souverain) et par les citoyens (ou sujets), et l'établissement de la relation entre les membres de ce corps politique. Le lien entre eux (État et citoyens) consisterait dans l'aliénation complète de l'individu, sa personnalité et ses biens pour l'État, et ensuite, dans la reconstruction de l'individu par l'État, avec la garantie de tout ce qui serait nécessaire pour assurer à chacun la jouissance égale des droits. C'est pourquoi le citoyen serait bien plus libre avant le Contrat qu'après. (BOUTMY, 1907, p. 124-125).

De même que la Déclaration, selon Boutmy, l'essence du contrat serait l'égalité de droits de tous les citoyens, le fondement de la loi dans la nécessité du maintien de l'isonomie entre eux et l'inéluctable généralité de la loi. Cela éliminerait toute idée de contradiction entre les Déclarations de droit, spécialement la Déclaration Française de 1789, et les principes du Contrat social et la philosophie de Rousseau (BOUTMY, 1907, p. 125).

La recherche de l'origine de la Déclaration Française de 1789 est le fondement pour la vision historique des droits humains. La Déclaration Française de 1789 ne doit pas être réduite à une simple copie des Déclarations américaines, et cela est fait par Jellinek quand il répond aux critiques de Boutmy. Un exemple de cela est le fait que la Constitution Française de 1789 et l'Américaine de 1776 sont complètement distinctes. De plus, la Déclaration de 1789 a été la première à étendre les droits reconnus à tous les êtres humains, différemment des Déclarations américaines qui protégeaient seulement ses citoyens.

Autres caractéristiques de la Déclaration Française de 1789 : a) transcendance - le préambule révèle ne pas avoir l'intention de faire un travail créatif ; b) universalisme - droits étendus à tous les êtres humains, indépendamment de la nationalité, la religion, l'ethnie etc. ; c) individualisme - seul l'individu est titulaire de droits reconnus (la Nation a été l'unique collectivité mentionnée dans la Déclaration) ; d) abstraction - les principes présentés (liberté, égalité, assurance juridique et droit à la propriété) n'ont pas de finalité prédéfinie, chacun 
les utilisant à son bon vouloir (MORANGE, 1982, p. 34). La lecture comparative de la Déclaration des Droits de l'Homme et du Citoyen de 1789 et de la Déclaration Universelle des Droits Humains de 1948 montre l'influence que le texte français a exercé sur celle-ci. Sans oublier que la DUDH contient des normes de droit international, alors que la Déclaration de 1789, en dépit de ses aspects de transcendance et d'universalité, découle de normes créées par un seul sujet. Les critiques à ces caractéristiques seront faites ultérieurement.

Gregorio Peces-Barba (1981, p. 169-253) signale La Declaración de los Derechos del Hombre y del Ciudadano de Georg Jellinek, comprenant la réponse à Émile Boutmy, comme étant un texte qui contribue à l'analyse historique de l'origine des droits humains en profondeur, concernant le niveau de formation de ses valeurs et principes éthiques. Il est important de ne pas confondre vision historique et argumentation historiciste.

\section{AUTRE DÉSACCORD AUTOUR DES DROITS HUMAINS}

Où nait la force des droits humains ? L'histoire des droits humains remonte au XVIIe siècle et, bien qu'il existe une asymétrie temporelle entre cette histoire et la récente codification de la dignité humaine dans le droit international, c'est en elle que les droits humains trouvent leur fondement (HABERMAS, 2012, p. 10). Les droits humains se rattachent à l'idée d'être humains jouissant de contextes minimalement dignes. La culture des droits humains s'est trouvée renforcée à partir de la reconstruction des États et des communautés après les crimes de masse (guerres mondiales, génocides etc). L'idée de droits humains peut servir de paramètre pour valoriser des actions ou des comportements. Les crimes de masse, par exemple, sont avant tout considérés crimes parce qu'ils violent essentiellement l'idée de droits humains. En d'autres termes, la raison/idée trouvée derrière les actes de violence en masse ne s'harmonise pas avec la notion d'expériences minimalement humaines.

La reconstruction des États et des communautés après les crimes de masse part de la perspective analytique qui s'écarte de la dichotomie « la guerre de tous contre tous »/ « la pure manipulation de populations pacifiques ». La raison attaquée par cette perspective est celle qui nie la possibilité de vie sociale commune parmi des êtres différents. Cette négation de l'humanité potentialise les crimes de masse (POULIGNY, 2007). La reconstruction de la paix doit prendre en compte comment la violence transforme la société, les effets post-génocide, les différentes mémoires et les représentations de la violence. Toutes ces idées harmonieuses ou discordantes affectent et modifient l'idée des droits humains.

Les êtres humains, les États, les collectivités d'État et les acteurs 
politiques jouent un rôle clef dans le processus de «percevoir » les mémoires des massacres commis dans l'histoire, sans négliger le fait que rappeler ou oublier n'est pas un processus linéaire. Les discours officiels peuvent promouvoir les conflits ou la construction de la paix, tout comme le fait la mémoire. Toutefois, dans la plupart des cas, ce processus de contraste n'est pas aussi évident en soi. Il y a des idéologies créées à partir d'un quantum arbitraire élevé qui a recours à divers moyens de camoufler l'incompatibilité avec l'idée de droits humains. Tout cela se produit y compris au sein même de la conception des droits humains.

Même si la dignité humaine n'était pas vraiment exprimée dans les premières déclarations, elle y était implicitement présente, dans le noyau des droits humains, alimentée par les injustices subies par les êtres humains dans de nombreux processus historiques. En raison de celle-ci, les droits humains sont indivisibles. Cependant, le fondement de la dignité humaine ne rend pas les droits humains moins abstraits. Les droits humains font partie de l'idéologie dominante de la société internationale, d'une grande partie des Etats et des communautés. L'abstraction de ces droits ne doit pas être l'objet d'une recherche sous un aspect terminatif négativiste. Elle doit prendre en compte les pratiques sociales qui les concernent. La question de l'universalité des droits humains illustre bien cette critique. D'une manière générale,

\section{[...] mientras para la crítica filosófica la universalidad es impugnada por su carácter ideal y abstracto, para la crítica política se la reputa nociva porque intenta allanar y desconocer las diferentes tradiciones políticas de las distintas culturas, en tanto que desde la crítica jurídica se insistirá en que la universalidad es imposible, al no existir un marco socioeconómico que permita satisfacer plenamente todos los derechos humanos a escala planetaria [...]. (PÉREZ LUÑO, 2002, p. 36).}

Même ainsi, il y a des points positifs à l'abstraction des droits humains. Pour Pérez Luño, les philosophes dits post-modernes qui ont émis des critiques au caractère idéal et abstrait des droits humains ne sont post-modernes qu'au sens chronologique et non pas qualitatif (PÉREZ LUÑO, 2002, p. 37-38). Face à la nécessité traditionnelle d'apporter un fondement philosophique aux droits humains - écartée par Rabossi -, Pérez Luño signale la création d'une synthèse de valeurs multinationales et multiculturelles qui rende possible la communication intersubjective, la solidarité et la paix. Il s'agit de trouver l'ethos universel.

Selon lui, laisser de côté l'ethos universel au profit d'un 
nationalisme radical est une absurdité logique et éthique. Se fondant sur Hume et Moore, Pérez Luño montre que, du point de vue logique, le nationalisme représente l'une des manifestations du raisonnement fallacieux naturaliste (Naturalistic Fallacy), car le discours nationaliste part toujours de diverses évidences de fait ; par exemple, les défis distincts qu'ont certains groupes ou personnes en raison de la couleur de la peau, des cheveux, des croyances et aptitudes (PÉREZ LUÑO, 2002, p. 39). Le respect des traditions politiques de cultures distinctes impose la nécessité de communication intersubjective, dans la mesure où le droit à la différence ne peut devenir un droit à l'indifférence (IMBERT apud PÉREZ LUÑO, 2002, p. 40).

Dans les critiques juridiques, il y a deux formes de traiter l'universel : comme universalité dans les droits humains et comme universalité des droits humains. La première (sens extensif et descriptif) renvoie à l'accueil des droits humains dans tous les ordres juridiques. La seconde (sens intensif et prescriptif) se demande si l'universalité est un élément inhérent ou constitutif du concept des droits humains. Pérz Luño ne trouve de sens que dans la critique juridique sur l'universalité dans les droits humains (2002, p. 44).

En fait, est inhérente aux droits humains l'universalité de leurs préceptes ; défendre le contraire serait soutenir quelque autre espèce de droit, sauf les droits humains. L'abstraction universelle fait partie de l'idée de droits humains et ne doit pas être analysée comme quelque chose de négatif. Cependant, quels droits doivent être considérés comme étant des droits humains? Il est vrai que la question de l'inhérence et de l'essentialité de certains préceptes contenus dans la DUDH a affaibli l'idée d'universalité. Il est important que les critiques de l'idéologie des droits humains soient confrontées aux pratiques sociales, afin d'identifier les idéologies arbitraires.

Outre les critiques déjà connues à chacune de ces argumentations, il y a des auteurs - comme par exemple l'Argentin Eduardo Rabossi - qui présentent les droits humains comme des faits du monde. La thèse défie les philosophes (et autres spécialistes des droits humains) en pensant la culture des droits humains comme quelque chose de créé par le monde post-Holocauste (post-crimes de masse), dont la violation ne ferait que renforcer son existence. La valorisation de comportements et la création de structures, programmes et politiques publiques font des droits humains des faits du monde. La création des Nations-Unies (1945) marque le phénomène des droits humains. La reconnaissance légale est nécessaire, mais insuffisante à la pleine jouissance des droits. Ainsi même, l'échec des argumentations ne parvient pas à les écarter comme faits du monde. Ils sont toujours là, composant les idéologies sociales.

Les arguments sont compatibles avec la pertinence de la connaissance historique (no historiciste) des droits humains, surtout à 
partir des révolutions bourgeoises américaine et française. La discussion entre Jellinek et Boutmy sur les influences de la Déclaration Française de 1789 apporte des points importants à cette vision historique des droits humains. La lecture comparative de la Déclaration des Droits de l'Homme et du Citoyen de 1789 et de la Déclaration Universelle des Droits Humains de 1948 montre l'influence que le texte français a exercé sur cette dernière. Dans les déclarations, les droits sont transcendantaux, universels, individuels et abstraits.

La Déclaration Française de 1789, influencée par le contrat social de Rousseau, ne peut se résumer à une simple copie des Déclarations américaines. Elle a été le premier texte à étendre à tout le monde (pas seulement au citoyen français) les droits reconnus. De cette origine à l'idéologie actuelle, la notion de droits humains a subi maintes transformations.

La discussion sur les droits humains et leurs caractéristiques est essentiellement un conflit d'idéologies. La théorisation sur la question de l'idéologie est diverse. Le premier aspect se présente dans les sens faible et fort de l'idéologie. Dans le sens faible, l'idéologie apparait comme source des idées, lieu où sont désignés les systèmes de croyances politiques et valeurs. Le sens fort correspond aux contributions de la critique marxiste de distorsion de la connaissance. Il est toutefois possible de visualiser des différences internes de l'idéologie-idéologies historiquement organiques et idéologies arbitraires. Il faut disqualifier les idéologies arbitraires par l'analyse critique, alors que les idéologies historiquement organiques construisent les champs d'avancées scientifiques dans lesquels les représentations de la réalité sont, même provisoirement, des validités. Bien que les idéologies dominantes ne soient pas toujours le reflet de la réalité sociale, les réduire comme synonyme de " fausse conscience » sépare la critique de l'idéologie de la recherche d'autonomie. En d'autres termes, l'impuissance est condamnée et le pouvoir de l'idéologie remise à un autre groupe social.

L'un des façons de parler de la négation des droits humains consiste à les rapporter aux régimes totalitaires (MORANGE, 1982, p. 66). À partir du XXe siècle, la philosophie des droits humains est devenu l'objet de critiques radicales. Le refus systématique des fondements des droits humains a renforcé les discours de supériorité (MORANGE, 1982, p. 60), à l'instar des régimes fasciste (supériorité absolue de l'État), nazi (supériorité de la race) et staliniste (supériorité idéologique d'une certaine classe). Le marxisme, dans la pratique, se fondait sur le pouvoir autoritaire qui s'appuyait sur une nouvelle couche sociale, sur une nouvelle classe (SCHWARTZENBERG, 1979, p. 91-92).

La création d'une nouvelle classe dominante est présentée par Claude Lefort (Éléments d'une critique de la bureaucratie), ainsi que par Milovan Djilas (La nouvelle classe dirigeante), par Marc Paillet 
(Marx contre Marx, La société technobureuacratique) etc. Quant au pouvoir autoritaire, il a établi la dictature sur le parti et sur l'État. Au lieu de disparaître, l'État a persisté dans tous ses aspects (justice, police, défense nationale, gestion de l'économie et.), s'appuyant sur trois piliers : sur l'appareil du parti (Stalin comme secrétaire-général) ; sur l'énorme bureaucratie de l'État (dirigisme et centralisme économique) et sur la police (qui préfabrique de nombreux procès politiques et exécute les expurgés) (SCHARWTZENBERG, 1979, p. 91). D'une manière générale, le totalitarisme crée, selon l'expression de Hannah Arendt, l'homme sans âme (MORANGE, 1982, p. 60). Le régime déconstruit la personnalité de l'être humain et le lance dans une personnalité juridique vide et manipulée selon le type de supériorité instaurée.

La question de l'existence des droits humains peut se référer à la justification morale (RABOSSI, 1990, p. 167). Les droits humains existeraient quand il y aurait reconnaissance sociale et promulgation légale. Une autre forme pour qu'existent les droits humains serait la nécessité de raisons morales justifiant ou fondant les exigences morales constitutives de la nature de ces droits, même si l'existence des droits humains ne dépend pas du succès de cette justification philosophique. L'échec d'une tentative de découverte des raisons signifierait simplement qu'il y a encore quelque chose à découvrir (RABOSSI, 1990, p. 67).

Obrad Savic présente l'article d'Eduardo Rabossi - Human Rights Naturalized -, ratifiant 1'argument selon lequel les philosophes doivent penser à la culture des droits humains comme quelque chose de nouveau, créé dans le monde post-crimes de masse. Pour Rabossi, des philosophes tel que Alan Gewirth auraient tort d'argumenter que les droits humains ne pourraient dépendre de faits historiques. Les changements dans le monde, ainsi que le phénomène des droits humains, ont transformé les efforts concernant l'argumentation du fondement des droits humains en quelque chose de « démodé » et de peu d'importance (SAVIC, 2013, p. 69).

Eduardo Rabossi se penche sur les arguments concernant la nécessité de l'argumentation du fondement, support ou justification rationnelle des droits humains (RABOSSI, 1990, p. 159-160). Les fondamentalistes, comme il les appelle, soutiennent que :l'argumentation morale des droits humains présuppose une contribution philosophique importante à l'existence de la théorie des droits humains ; les droits humains sont du type droits moraux ; les droits humains sont formés à partir d'un principe moral ou d'un ensemble de principes moraux (RABOSSI, 1990, p. 160). Rabossi observe que ces argumentations ne s'ajustent pas aux faits et qu'elles ne sont pas suffisamment persuasives.

Les droits humains seraient des " faits du monde ». La constatation des violations de droits humains peut attrister, susciter leur refus ou l'expression d'une opinion à leur sujet etc. En outre, 
l'existence de groupes et de mouvements défenseurs des droits humains est reconnue; face à cela, il est possible de les critiquer, de les rejoindre etc. Des comportements sont valorisés et les droits humains sont utilisés comme bannières. Voilá des exemples qui, selon Rabossi, font des droits humains des faits du monde. Ils forment notre vision du monde, puisqu'ils guident la façon dont on valorise des aspects importants de la vie (personnels, sociaux et politiques). Pour l'auteur argentin, « [...] il existe une florissante culture des droits humains dans le monde. Nous en faisons partie. Nous sommes immergés en elle » (RABOSSI, 1990, p. 159). La création des Nations-Unies (1945) a été la principale borne du phénomène des droits humains, vu qu'elle est apparue avec l'intention de créer une communauté mondiale.

L'Organisation des Nations-Unies réglemente les relations amicales entre les États fondées sur l'égalité et l'autodétermination des peuples, et elle prend des mesures pour renforcer la paix. En plus de déclarer des principes, la Charte de l'ONU crée des organes (Assemblée Générale, Secrétariat Général, Conseil de Sécurité etc.) ayant leurs propres attributions. Le souci de la Charte envers les droits humains découle de l'engagement assumé par les États-membres de coopérer avec l'ONU. La Déclaration Universelle des Droits Humains (1948) a entamé un processus complexe de codification des droits humains. Le Pacte International sur les Droits Économiques, Sociaux et Culturels et le Pacte International sur les Droits Civils et Politiques sont les instruments légaux de base de ce processus. La reconnaissance légale des droits humains ne doit pas être considérée comme une simple liste de droits, car elle crée aussi des entités, des organes, des commissions, des groupes, des agences, des comités etc. Dotés d'attributions et de juridiction. Il y a des structures semblables dans le cadre régional (Union Européenne - UE, Organisation des États Américains - OEA, Union Africaine - UA, Ligue Arabe - LA ) (RABOSSI, 1990, p. 163).

Afin de tracer les contours des droits humains, Eduardo Rabossi partage les axes en "synchroniques 》 et " diachroniques ». Les premiers sont : a) le système normatif positif (types de normes, types de droits) ; b) le système institutionnel positif (agences et cours) ; c) le système informel ; d) les forces idéologiques et politiques opératrices dans le système et sur le système ; e) le système universel face aux systèmes régionaux ; f) la fonctionnalité de tout le système ; g) les problèmes légaux et conceptuels qui affectent le système normatif (lacunes, incohérences, " modifications » conceptuelles). Dans les éléments diachroniques, se trouvent : a) l'évolution des droits récemment positivés (à partir de 1945) ; b) l'apparition et les possibles solutions de certains problèmes mondiaux vexatoires (décolonisation, discrimination, apartheid, autodétermination, désastres écologiques, éducation, faim etc.) ; c) l'évolution possible de tout le système les 
années à venir ; d) les perspectives d'une communauté mondiale pacifique (RABOSSI, 1990, p. 163).

\section{Conclusion}

Face à tout cela, quelle est la transcendance du phénomène des droits humains?

D'un point de vue légal : - la promulgation légale des droits humains : sa positivation ; - la reconnaissance légale ( positive) des personnes individuelles (et de certains groupes) en tant que sujets propres de la loi internationale ; - l'établissement d'un système d'inspection sur les États ( concernant les violations des droits humains) ; - la création d'agences internationales ayant leur propre juridiction ; - l'existence de sanctions (dénonciation publique, blocus économique, "pression » politique etc.) ; - le fonctionnement d'une confédération mondiale ; la création d'un système normatif positif ayant divers niveaux de généralisation.

D'un point de vue politique : - la modification substantielle de l'idée traditionnelle de souveraineté d'État comme étant illimitée et libre de tout contrôle externe ; - une avancée progressive vers la construction réelle d'une communauté mondiale ; - une avancée graduelle vers un contrôle international des relations internationales (politiques et économiques); - la " diffusion » de l'idée "vivre dans une communauté mondiale $»$.

D'un point de vue théorique : - la reconnaissance consensuelle d'une série de fins et de valeurs universelles ; - l'affirmation, par le biais d'une promulgation légale, de cette série de valeurs et de fins ; la « confluence » de tendances opposées d'une tradition humanistique commune (RABOSSI, 1990, p. 164-165).

La reconnaissance légale est condition nécessaire, mais non suffisante, à la pleine jouissance des droits (RABOSSI, 1990, p. 171). Même ainsi, de nos jours il n'est pas nécessaire de recourir à des arguments moraux comme uniques raisons pour dénoncer l'illégitimité de quelque législation et de certaines décisions d'État. L'ouverture à des discussions relatives aux meilleures méthodes pour organiser les sociétés politiques et civiles est le signe essentiel à la promulgation légale des droits humains. La question de la nécessité d'argumentation pour le fondement des droits humains est une thèse sans intérêt pour le phénomène des droits humains. Selon Rabossi, il est possible que les fondamentalistes avancent dans un fait du monde dépassé. Cette posture ouvre un espace à un autre champ de la perspicacité philosophique : a) le dessin d'une borne opératoire conceptuelle ayant pour finalité de décrire et de valoriser le phénomène des droits humains ; b) l'aide pour éclaircir le contenu des mots-clés, les difficultés normatives et les 
problèmes de création; c) l'élaboration de l'importance philosophique du « point de vue théorique » etc. (RABOSSI, 1990, p. 174).

Les débats concernant les méthodes politiques conflictuelles au moment d'organiser les sociétés, et la distribution des libertés et des biens sont importants au sein du phénomène des droits humains. L'abstraction des droits humains, tout spécialement la caractéristique universelle, ne doit pas être paralysée devant la notion péjorative d'idéologie. Il est indispensable de vérifier ces contenus au regard des pratiques sociales et d'impulser le processus critique des idées. En ce qui concerne l'universalité, par exemple, on peut partir de la distinction utile entre universalité dans les droits humains et universalité des droits humains. L'universalité étant inhérente aux droits humains, encore faut-il centrer les efforts sur la tâche d'identification des idéologies arbitraires, négatrices d'humanité, et repenser la signification de l'extension de l'accueil de ces droits dans les ordres juridiques, en les confrontant constamment aux pratiques sociales. Même lorsqu'ils sont moralement fondés, les droits humains ont besoin d'être spécifiés et clarifiés démocratiquement (HABERMAS, 2012, p. 18).

Les droits humains qui devront prévaloir représenteront-ils toujours la protection d'un attribut universel présent en tout être humain ? Ou bien ces standards devront-ils dépendre de la culture ou du groupe social de la personne humaine ? Tandis que certains manifestent la préoccupation concernant la limitation des recours à la critique et à la justification de la diversité des codes moraux, d'autres jugent illégitime (et improbable) la recherche de normes extensives à tous qui que ce soit, où que ce soit. Les normes morales ne s'acquièrent qu'au sein de la structure sociale, autrement dit, dans l'ensemble particulier des restrictions physiques et sociales ? Et les raisons qu'elles ont pour justifier leurs normes ne seront-elles impositives qu'à ceux qui partagent la même condition ? Nombreuses sont les questions concernant l'étendue des droits humains.

\section{RÉFÉRENCES}

ALEXY, Robert. Tres escritos sobre los derechos fundamentales y la teoría de los principios. Traducción de Carlos Bernal Pulido. Colombia: Digiprint Editores, 2003.

. Direitos fundamentais, ponderação e racionalidade. Revista de direito privado - RT, São Paulo, nº 24, p. 334-344. Outubro/Dezembro, 2005.

Tres escritos sobre los derechos fundamentales y la teoría de los principios. Traducción de Carlos Bernal Pulido. Colombia: Digiprint Editores, 2003. 
- Teoria dos direitos fundamentais. Tradução de Virgílio

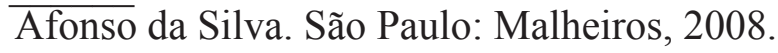

ARBUET-VIGNALI, Heber. Peligrosos prejuicios sobre soberanía. In: Anuario de derecho constitucional latinoamericano. Tomo I. Uruguay: Fundación Konrad-Adenauer, 2006, p. 65-104.

ARENDT, Hannah. La crise de la culture: huit exercices de pensée politique. Traduit de l'anglais sous la direction de Patrick Lévy. Trebaseleghe: Éditions Gallimard, 2012.

. Origens do totalitarismo: Antissemitismo, imperialismo, totalitarismo. Traduit par Roberto Raposo. São Paulo: Companhia das Letras, 2012.

. La condición humana. Traducido por Ramón Gil Novales. Buenos Aires: Paidós, 2012.

A promessa da política. Traduit par Pedro Jorgensen Jr. Rio de Janeiro: 2008. Paidós, 1997.

¿Qué es la política? Traducido por Rosa Sala Carbó. Barcelona:

ATIENZA RODRÍGUEZ, Manuel. Marx y los derechos humanos. Lima: Palestra, 2008.

BOBBIO, Norberto. Teoria do ordenamento jurídico. Tradução de Maria Celeste Cordeiro Leite dos Santos. 6. ed. Brasília: Editora da Universidade de Brasília, 1995.

. Teoria da norma jurídica. Tradução de Fernando Pavan Baptista e Ariani Bueno Sudatti. 2. ed. São Paulo: EDIPRO, 2003.

BOSON, Gerson de Brito Mello. O homem como sujeito de direito internacional. Belo Horizonte: [S.n.], 1951.

BOUTMY, Émile. La déclaration des droits de l'homme et du citoyen et M. Jellinek. In: Études politique. Paris: Armand Colin, 1907, p. 119-182. COLLOQUE DE LILLE. La juridictionnalisation du droit international. Paris : A. Pedone, 2003.

FERNÁNDEZ GARCÍA, Eusebio. El problema del fundamento de los derechos. Anuario de derechos humanos - 1981. Madrid: Instituto de Derechos Humanos de la Facultad de derecho, Universidad Complutense Madrid-3, 1981, p. 77 - 112.

- Dignidad humana y ciudadanía cosmopolita. Madrid: Instituto de derechos humanos Bartolomé de las Casa Universidad Carlos III de Madrid/Dykinson, 2001.

FONSECA, José Roberto Franco da. Geopolítica e direito internacional. 
Revista da Faculdade de Direito. São Paulo: Universidade de São Paulo, p. 315-329 v. 91, 1996.

HABERMAS, Jürgen. Sobre a constituição da Europa: um ensaio. Traduit par Denilson Luis Werle, Luiz Repa e Rúrion Melo. São Paulo: Unesp, 2012.

HERRERA FLORES, Joaquín. Los derechos humanos como productos culturales: crítica del humanismo abstracto. Madrid: Catarata, 2005.

JELLINEK, Georg. Teoría general del Estado. Tradiccíon de Fernando de los Ríos. México: FCE, 2000.

. La Declaración de los Derechos del Hombre e del Ciudadano. Traducción de Adolfo Posada. México: Universidad Nacional Autónoma de México, 2000.

JULLIEN, François. De l'universel: de l'uniforme, du commun et du dialogue entre les cultures. Paris: Fayard, 2008.

. Os direitos do homem são mesmo universais? Le Monde Diplomatique Brasil. São Paulo, fev. 2008, p. 30-31.

. O diálogo entre culturas: do universal ao multiculturalismo. Traduit par André Telles. Rio de Janeiro: Zahar, 2009.

KYMLICKA, Will. Multiculturalismo Liberal e Direitos Humanos. In: Daniel Sarmento, Daniela Ikawa e Flávia Piovesan (org.). Igualdade, diferença e direitos humanos. Rio de Janeiro: Lumen Juris, 2008.

LAFER, Celso. A internacionalização dos direitos humanos: Constituição, racismo e relações internacionais. São Paulo: Manole, 2005. 1982.

. Paradoxos e possibilidades. Rio de Janeiro: Nova Fronteira,

MBAYA, Etienne Richard. Relations between individual and collective human rights: the problema of rights of peoples. In: Law and State. vol. 46, p. 7-23.

MORANGE, Jean. Droits de l'homme et libertés publiques. Paris: PUF, 1982.

PARLETT, Kate. The individual in the international legal system: continuity and change in international law. Cambridge: Cambridge University Press, 2010.

PECES-BARBA, Gregorio. Derechos Fundamentales en la cultura jurídica española. Anuario de derechos humanos - 1981. Madrid: Instituto de Derechos Humanos de la Facultad de derecho, Universidad 
Complutense Madrid-3, 1981, p.169-253.

. La dignidad de la persona desde la filosofía del derecho. Madrid: Dykinson, 2003.

PÉREZ LUÑO, Antonio E. Temas claves de la Constitución Española: Los derechos fundamentales. 8. ed. Madrid: Tecnos, 2004.

. La universalidad de los derechos humanos y el Estado constitucional. Bogotá: Universidad Externado de Colombia, 2002.

PRONER, Carol. Os direitos humanos e seus paradoxos: análise do sistema americano de proteção. Porto Alegre: Fabris, 2002.

RABOSSI, Eduardo. La teoría de los derechos humanos naturalizada. Revista del Centro de Estudios Constitucionales, ISSN 0214-6185, $\mathrm{n}^{\circ}$. 5, 1990 .

RADBRUCH, Gustav. Cinco minutos de filosofia do direito. In: Filosofia do Direito. Tradução de L. C. de Moncada, 6. ed. Coimbra: Armênio Amado Editor, 1997. p. 415-418.

. Arbitrariedad Legal y Derecho Supralegal. Buenos Aires: Abeledo - Perrot, 1962.

SAVIĆ, Obrad. The politics of human rights. Disponible sur : $<$ http:// books.google.com.br>. Acèss le: 22/01/2013.

SAYED, Bachir Mustafa. Los procesos de autodeterminación de los pueblos coloniales. Universidad de Barcelona, 1997. Disponible sur : $<$ http://www.arso.org/bachir1.htm>. Acèss le : 29/01/2013.

SCHWARTZENBERG, Roger-Gérard. Sociologia política: elemento de ciência política. Traduit par Domingos Mascarenhas. São Paulo: Difel, 1979.

SORTO, Fredys Orlando. Considerações sobre a primeira Corte internacional de Justiça. In: Solução e prevenção de litígios internacionais. São Paulo: NECIN-CAPES; Porto Alegre: Livraria do Advogado, p. 275-316, 1999.

.A Doutrina Bush das guerras preventivas e o Sistema das Nações Unidas. In: MERCADANTE, Araminta de Azevedo; MAGALHÃ̃ES, José Carlos (Orgs.). Reflexões sobre os 60 anos da ONU. Ijuí: Unijuí, 2005, p. 130-163.

. A Declaração Universal dos Direitos Humanos no sexagésimo aniversário. Verba Juris: Anuário de Pós-Graduação em Direito, João Pessoa, ano 7, n. 7, p. 9-34, jan./dez. 2008.

. O projeto jurídico de cidadania universal: reflexões à luz do $\overline{\text { direito }}$ de liberdade. Anuário hispano-luso-americano de derecho 
internacional, Madrid, vol. 20, p. 103-126, ene./dic. 2011.

. A condição da pessoa humana no Projeto de Código de Direito Internacional Público de Epitácio Pessoa. In: Epitácio Pessoa e a codificação do direito internacional. Porto Alegre: Sergio Antonio Fabris, 2013, p. 133-155.

SUDRE, Frédéric. Droit européen et international des droits de l'homme. 11. ed. Paris: Presses Universitaires de France, 2012.

TRINDADE, Antônio Augusto Cançado. Princípios do direito internacional contemporâneo. Brasília: Universidade de Brasília, 1981.

. Desafios para a Efetiva Proteção Internacional dos Direitos Humanos. In: MEDEIROS, Antônio Paulo Cachapuz de (org.). Desafios do Direito Internacional Contemporâneo. Brasília: FUNAG, 2007, p. 207-321.

VALLADÃO, Haroldo. Democratização e socialização do Direito Internacional: Os impactos latino-americanos e afri-asiáticos. Rio de Janeiro: Livraria José Olympio, 1961. 